\title{
CONTINUITY OF THE MEASURE OF THE SPECTRUM FOR DISCRETE QUASIPERIODIC OPERATORS
}

\author{
S. YA. Jitomirskaya AND I. V. KRASOVSKY
}

\begin{abstract}
We study discrete Schrödinger operators $\left(H_{\alpha, \theta} \psi\right)(n)=\psi(n-1)+$ $\psi(n+1)+f(\alpha n+\theta) \psi(n)$ on $l^{2}(Z)$, where $f(x)$ is a real analytic periodic function of period 1. We prove a general theorem relating the measure of the spectrum of $H_{\alpha, \theta}$ to the measures of the spectra of its canonical rational approximants under the condition that the Lyapunov exponents of $H_{\alpha, \theta}$ are positive. For the almost Mathieu operator $(f(x)=2 \lambda \cos 2 \pi x)$ it follows that the measure of the spectrum is equal to $4|1-| \lambda||$ for all real $\theta, \lambda \neq \pm 1$, and all irrational $\alpha$.
\end{abstract}

\section{Introduction}

Consider quasiperiodic operators acting on $l^{2}(\mathbb{Z})$ and given by:

$$
\left(H_{\alpha, \theta} \psi\right)(n)=\psi(n-1)+\psi(n+1)+f(\alpha n+\theta) \psi(n), \quad n=\ldots,-1,0,1, \ldots,
$$

where $f(x)$ is a real analytic periodic function of period 1. Denote by $S(\alpha, \theta)$ the spectrum of $H_{\alpha, \theta}$. For rational $\alpha=p / q$ the spectrum consists of at most $q$ intervals. Let $S(\alpha)=\bigcup_{\theta \in \mathbb{R}} S(\alpha, \theta)$. Note that for irrational $\alpha$ the spectrum of $H$ (as a set) is independent of $\theta$ (see, e.g., [7]), and therefore $S(\alpha, \theta)=S(\alpha)$. In this paper we study continuity in $\alpha$ of $S(\alpha)$ and its measure. For sets, we will use $|\cdot|$ to denote the Lebesgue measure.

The fact that various quantities could be easier to analyse and sometimes are even computable for periodic operators, $H_{p / q, \theta}$, makes results on continuity in $\alpha$ particularly important. For example, the Aubry-Andre conjecture on the measure of the spectrum [1] states that for the almost Mathieu operator given by (1.1) with $f(\theta)=2 \lambda \cos 2 \pi \theta$, for irrational $\alpha$ and all real $\lambda, \theta$ there is an equality

$$
\left|S_{\lambda}(\alpha, \theta)\right|=4|1-| \lambda|| \text {. }
$$

Avron,van Mouche, Simon [3] proved that, for $|\lambda| \neq 1,\left|S_{\lambda}\left(p_{n} / q_{n}\right)\right| \rightarrow 4|1-| \lambda||$ as $q_{n} \rightarrow \infty$, and Last [17] established this fact for $|\lambda|=1$. Given these theorems, the proof of the Aubry-Andre conjecture was reduced to a continuity result.

The continuity in $\alpha$ of $S(\alpha)$ was proven in $[2,8]$. Continuity of the measure of the spectrum is a more delicate issue, since, in particular, $|S(\alpha)|$ can be discontinuous at rational $\alpha$. Establishing continuity at irrational $\alpha$ requires

Received July 11, 2001.

Revised version received December 5, 2001. 
quantitative estimates on the continuity of the spectrum. The first such result, namely the Hölder- $\frac{1}{3}$ continuity was proved in [6]. That argument was improved to the Hölder-1/2 continuity (for arbitrary $f \in C^{1}$ ) in [3] and subsequently used in $[16,17]$ to establish (1.2) for the almost Mathieu operator for $\alpha$ with unbounded coefficients of continued fraction expansion, therefore proving the Aubry-Andre conjecture for a.e. (but not all) $\alpha$. It was essentially argued in [3] that Hölder continuity of any order larger than $1 / 2$ would imply the desired continuity property of the measure of the spectrum for all $\alpha$. Such continuity (more precisely, almost Lipschitz continuity, as in Theorem 3) was proved in [13] for the almost Mathieu operator with $|\lambda|>14.5$ (or dual regime), using exponential localization for Diophantine frequencies and delicate analysis of generalized eigenfunctions (positions of resonances and behavior between them).

In the present paper we prove almost Lipschitz continuity of the spectrum for operators (1.1) with arbitrary analytic $f$ under the assumption of positivity of the Lyapunov exponents. We would like to note that, unlike in [13], we do not rely on the theorem on exponential localization, and the present paper is essentially self-contained. In fact, the localization theorem for general analytic potentials, proved in [4], establishes pure point spectrum for a.e. $\alpha$ without explicit Diophantine control, and therefore would not be applicable for our purposes. On the other hand, we would like to mention that, as the proof below shows, continuity of the spectrum requires simpler arguments than localization.

Let $M_{n}$ be the transfer-matrix of $H \psi=E \psi$ :

$$
\left(\begin{array}{c}
\psi(n+1) \\
\psi(n)
\end{array}\right)=M_{n}(\theta, E)\left(\begin{array}{c}
\psi(n) \\
\psi(n-1)
\end{array}\right)
$$

Then

$$
M_{n}(\theta, E)=\left(\begin{array}{cc}
E-f(\alpha n+\theta) & -1 \\
1 & 0
\end{array}\right), \quad n=\ldots,-1,0,1, \ldots
$$

Let $T_{n}(\theta, E)=M_{n-1}(\theta, E) \cdots M_{1}(\theta, E) M_{0}(\theta, E)$ be the $n$-step transfer-matrix. The Lyapunov exponent $\gamma(E, \alpha)$ of the family (1.1) is defined as follows:

$$
\gamma(E, \alpha) \equiv \lim _{n \rightarrow \infty} \frac{1}{n} \int_{0}^{1} \ln \left\|T_{n}(\theta, E)\right\| d \theta=\inf _{n} \frac{1}{n} \int_{0}^{1} \ln \left\|T_{n}(\theta, E)\right\| d \theta .
$$

It is well defined by (1.3) by Kingman's subadditive ergodic theorem (see, e.g., [7]). Note that $\operatorname{det} M_{n}(\theta, E)=1$ whence it follows that $\gamma(E, \alpha) \geq 0$.

Our main result is the following:

Theorem 1. Let $\gamma(E, \alpha)>0$ for Lebesgue a.e. E. Then for any real $\theta$,

$$
|S(\alpha, \theta)|=\lim _{n \rightarrow \infty}\left|S\left(\frac{p_{n}}{q_{n}}\right)\right|,
$$

where $p_{n} / q_{n}$ is the sequence of canonical rational approximants to $\alpha$.

\section{Remarks.}

1. Note that a.e. positivity of $\gamma$ implies that $\alpha$ is irrational. 
2. For $\alpha$ whose coefficients of the continued fraction expansion form an unbounded sequence, the condition $\gamma(E, \alpha)>0$ is redundant. The result of Theorem 1 follows in this case (by a simple generalization of the argument in [16]) from the Hölder-1/2 continuity of the spectrum established in [3]. For $\alpha$ 's whose continued fraction coefficients form a bounded sequence (henceforth, we denote the set of such $\alpha$ by $\Omega$ ) this continuity is not sufficient to imply Theorem 1 . For such $\alpha$ we prove the theorem by first establishing the almost-Lipschitz continuity of the spectrum for which, in turn, we use positivity of the Lyapunov exponent.

3. Note that it is not clear apriori that the limit above exists.

4. While our proof can be easily adapted for arbitrary $\alpha$ we, for the reason above, concentrate only on the case $\alpha \in \Omega$, which slightly simplifies certain arguments.

We would like to add that recently a number of remarkable properties of quasiperiodic operators with analytic potentials have been established based on the positivity of the Lyapunov exponents $[12,4,14,10]$. Theorem 1 , therefore, adds to the collection of general corollaries of positive Lyapunov exponents for analytic potentials.

For the almost Mathieu operator Theorem 1 immediately implies:

Theorem 2. For every $\lambda, \theta \in \mathbb{R},|\lambda| \neq 1$, and every irrational $\alpha$, the measure of the spectrum of the almost Mathieu operator $H_{\lambda, \alpha, \theta}$ is equal to $4|1-| \lambda||$.

This improves some results of $[16,13]$ and establishes the Aubry-Andre conjecture on the measure of the spectrum for all values of parameters in the noncritical case. For the critical case, $|\lambda|=1$, zero measure of the spectrum is known for the full measure set of $\alpha[11,17]$, however extending it to all irrational $\alpha$ remains an open problem.

Proof. It was shown in [3] that on a sequence of rational approximants $p_{n} / q_{n}$ to any real $\alpha$ we have that $\left|S_{\lambda}\left(p_{n} / q_{n}\right)\right|(|\lambda| \neq 1)$ converges to $4|1-| \lambda||$. Assume $\lambda>0$ as the case $\lambda<0$ is easily obtained by the transform $\theta \rightarrow \theta+1 / 2$ and the case $\lambda=0$ is trivial. We further assume $\lambda>1$ as the case $0<\lambda<1$ can than be analyzed using duality [1,2]. For $\lambda>1$, it is known (e.g., [7]) that $\gamma(E, \alpha)>0$ for all real $E$ and irrational $\alpha$, which concludes the proof in view of Theorem 1.

In Section 2 we establish almost Lipschitz continuity of the spectrum and in Section 3 we complete the proof of Theorem 1.

\section{Continuity of the spectrum}

In what follows we shall often omit the indices $\alpha, \theta$, or $E$ from the notation. Denote the basis in which $H$ has the tridiagonal structure (1.1) by $\left\{e_{i}\right\}_{i=-\infty}^{\infty}$. Let $(E-H)_{\left[x_{1}, x_{2}\right]}$ be the operator $E-H$ restricted to the subspace spanned by $\left\{e_{i}\right\}_{i=x_{1}}^{x_{2}}$. Set $k=x_{2}+1-x_{1}$. Consider the polynomial in $E$ :

$$
\widetilde{P}_{k}(\alpha, \theta, E)=\operatorname{det}\left((E-H)_{[0, k-1]}\right), \quad \widetilde{P}_{0}=1 .
$$


Let $f_{k}$ be the Fourier truncation of $f(\theta)$ :

$$
f_{k}(\theta)=\sum_{j=-k^{2}}^{k^{2}} \hat{f}_{j} e^{2 \pi i j \theta}
$$

As $f(\theta)$ is an analytic function, its Fourier coefficients $\hat{f}_{j}$ are of order $e^{-c j}$. Hence $\left|f(\theta)-f_{k}(\theta)\right|<\exp \left(-d k^{2}\right)$, where $d>0$ depends on $f(\theta)$, for $k$ sufficiently large. Set $z=\exp (2 \pi i \theta)$. Let $H_{k}$ be the operator $H$ with $f(\theta)$ replaced by $f_{k}(\theta)$ and consider

$$
P_{k}(\alpha, z, E)=z^{k^{3}} \operatorname{det}\left(\left(E-H_{k}\right)_{[0, k-1]}\right) .
$$

Obviously, $P_{k}(z)$ is a polynomial of degree $2 k^{3}$ in $z$. Using induction for the three-term recurrence relation (which connects $\widetilde{P}_{n}, \widetilde{P}_{n-1}$, and $\widetilde{P}_{n-2}$ ) and boundedness of $f(\theta)$, we obtain for all $z,|z|=1$, and $E$ in a bounded set:

$$
\left|P_{k}\right|-e^{-d k^{2}}<\left|\widetilde{P}_{k}\right|<\left|P_{k}\right|+e^{-d k^{2}}, \quad\left|P_{k}\right|<e^{D k},
$$

where $D<\infty, d$ is somewhat decreased from the prior value but still positive, and $k$ is larger than some $k_{1}$.

We shall now obtain the upper and lower bounds on $\left|P_{k}(z, E)\right|$ which we use later to establish the continuity properties of the spectrum.

Lemma 1. (Estimate of $\left|P_{k}(z, E)\right|$ from below) For every $\alpha \in \Omega, E \in S, \varepsilon^{\prime}>0$ and at least one integer $k$ out of each three consecutive integers $s, s+1, s+2$, $s>k_{1}$, there exists $C\left(\varepsilon^{\prime}, \alpha\right)$ such that in any interval of length $C k^{3}$ there is an integer $m$ such that $\left|P_{k}\left(e^{2 \pi i(\theta+m \alpha)}, E\right)\right| \geq e^{\left(\gamma(E)-\varepsilon^{\prime}\right) k}$. The constant $C$ is independent of $E$.

Remark. This statement, appropriately adjusted, holds under any Diophantine condition on $\alpha$.

Proof. Since, as is easy to verify,

$$
T_{k}(\theta, E)=\left(\begin{array}{cc}
\widetilde{P}_{k}(\theta) & -\widetilde{P}_{k-1}(\theta+\alpha) \\
\widetilde{P}_{k-1}(\theta) & -\widetilde{P}_{k-2}(\theta+\alpha)
\end{array}\right), \quad k \geq 2,
$$

we can rewrite (1.3) in the form

$$
\gamma(E)=\inf _{k} \frac{1}{k} \int_{0}^{1} \ln \mu_{k}(\theta) d \theta
$$

where $\mu_{k}(\theta)=\max \left\{\left|P_{k}(\theta)\right|,\left|P_{k-1}(\theta)\right|,\left|P_{k-1}(\theta+\alpha)\right|,\left|P_{k-2}(\theta+\alpha)\right|\right\}$. Let $B_{k}=$ $\left\{\theta \in[0,1): \mu_{k}(\theta) \geq e^{\left(\gamma(E)-\varepsilon^{\prime}\right) k}\right\}$. Then, using (2.7) and (2.8), we obtain

$$
k \gamma(E) \leq \int_{0}^{1} \ln \mu_{k}(\theta) d \theta=\int_{B_{k}}+\int_{[0,1) \backslash B_{k}} \leq\left|B_{k}\right| k D+\left(1-\left|B_{k}\right|\right)\left(\gamma(E)-\varepsilon^{\prime}\right) k .
$$

Therefore, $\left|B_{k}\right| \geq \varepsilon^{\prime} /\left(D-\gamma(E)+\varepsilon^{\prime}\right) \geq 3 c_{1}\left(\varepsilon^{\prime}\right)$ for all $\mathrm{E}$ in any bounded set (in particular, the spectrum of $H$.) Hence among each three consecutive indices 
$s, s+1, s+2>k_{1}$, there is at least one, denote it $k$, such that

$$
\left|N_{k}:=\left\{\theta \in[0,1):\left|P_{k}\left(e^{2 \pi i \theta}\right)\right| \geq e^{\left(\gamma(E)-\varepsilon^{\prime}\right) k}\right\}\right| \geq c_{1}\left(\varepsilon^{\prime}\right)>0
$$

Denote by $M$ the set of all such $k$.

Since $P_{k}$ is a polynomial of degree $2 k^{3}$ in $e^{2 \pi i \theta}$, the set $N_{k}$ consists of less than $9 k^{3}$ intervals. Let $J$ be the interval in $N_{k}$ with the maximum length. We have

$$
|J| \geq \frac{c_{1}}{9 k^{3}}
$$

It follows from the Diophantine properties of $\Omega$ that $\theta+\operatorname{m\alpha }(\bmod 1) \in J$ for at least one $m \in I$, where $I$ is any interval of length $C k^{3}$. For reader's convenience we give a simple argument for the above.

Let $\alpha \in[0,1)$ be an irrational and consider the continued fraction expansion $\alpha=\left[0 ; a_{1}, a_{2}, \ldots\right]$, where $a_{i}$ are positive integers. Recall (e.g.,[15]) that the convergents $p_{n} / q_{n}=\left[0 ; a_{1}, a_{2}, \ldots, a_{n}\right]$ have the properties

$$
\alpha=\frac{p_{n}}{q_{n}}+\frac{\widetilde{\delta}}{q_{n} q_{n+1}}, \quad|\widetilde{\delta}|<1, \quad q_{n}=a_{n} q_{n-1}+q_{n-2}, \quad n=3,4, \ldots
$$

and the greatest common divisor $\operatorname{gcd}\left(p_{n}, q_{n}\right)=1$.

For a fixed $n$, consider the set $\theta_{j}=\theta+\alpha j(\bmod 1), j=0,1, \ldots, q_{n}-1$. Let $\theta_{j}$ and $\theta_{j^{\prime}}$ be nearest neighbors. We have, using (2.10),

$$
\left|\alpha j-\frac{j p_{n}}{q_{n}}\right|=\left|\frac{j \widetilde{\delta}}{q_{n} q_{n+1}}\right|<\frac{j}{q_{n} q_{n+1}}<\frac{1}{q_{n}} .
$$

Since the points $\frac{j p_{n}}{q_{n}}(\bmod 1), j=0,1, \ldots, q_{n}-1$ are the same as $\frac{j}{q_{n}}(\bmod 1)$, $j=0,1, \ldots, q_{n}-1$, up to the ordering, we have, using (2.11):

$$
\max \left|\theta_{j}-\theta_{j^{\prime}}\right|<\frac{3}{q_{n}}, \quad j=1,2, \ldots, q_{n} .
$$

Therefore, if $c_{1} / 9 k^{3} \geq 3 / q_{n}$, there exists $0 \leq m \leq q_{n}-1$ such that $\theta_{m} \in J$. Accordingly, choose $n$ so that

$$
q_{n-1}<\frac{27 k^{3}}{c_{1}} \leq q_{n}
$$

For $\alpha \in \Omega$ we have that $a_{n} \leq B(\alpha)$ for all $n$. Then

$$
q_{n}=a_{n} q_{n-1}+q_{n-2}<2 B(\alpha) q_{n-1}<C k^{3} .
$$

where $C=60 B(\alpha) / c_{1}$. Therefore, at one of any $q_{n}$ (as defined by (2.13)) of the points $\theta_{m}$ with consecutive indices we have the needed estimate for $P_{k}\left(e^{2 \pi i \theta}\right)$. Inequality (2.14) implies the statement of the Lemma.

Lemma 2. (Estimate of $\left|P_{k}(z, E)\right|$ from above) For any $\delta, \varepsilon>0$, there exists a set $F(\delta, \varepsilon) \subset S$ such that $|F(\delta, \varepsilon)|<\delta$ and for any $\alpha \in \Omega, E \in S \backslash F(\delta, \varepsilon)$, and sufficiently large $k\left(k>k_{2}(\delta, \alpha, \varepsilon)\right)$ we have $\left|P_{k}(z, E)\right| \leq e^{(\gamma(E)+\varepsilon) k}$. The constant $k_{2}$ is independent of $z$ and $E$. 
Remark. Lemma 2 actually holds uniformly, for all $E$ in a bounded set, [5]. However, this result requires a relatively complicated argument, while a nonuniform statement above follows immediately from a general theorem on uniquely ergodic dynamical systems and is sufficient for our purposes.

Proof. It is proved in [9] that for any continuous subadditive cocycle $f_{n}$ on a uniquely ergodic system, $\lim \sup \frac{1}{n} f_{n}(x) \leq \lim \frac{1}{n} \int f_{n} d \mu(x)$ uniformly in $x$. Therefore, for large $k$ we have $\left|P_{k}(z)\right|<e^{\left(\gamma_{k}(E, \alpha)+\varepsilon\right) k}$ for all $|z|=1$.

Let $D_{n}=\left\{E \in S: \forall k>n \forall|z|=1,\left|P_{k}(z, E)\right| \leq e^{(\gamma(E)+\varepsilon) k}\right\}$. Then we have $\left|S \backslash D_{n}\right| \rightarrow 0$ as $n \rightarrow \infty$, which completes the proof of Lemma 2 .

We are now ready to formulate the result about continuity of the spectrum.

Theorem 3. Suppose $\alpha \in \Omega$ and $\gamma(E, \alpha)>0$ for Lebesgue a.e. E. Then for any $\delta>0$, there exists a set $A(\delta) \subset S(\alpha)$ such that $|A(\delta)|<\delta$ and for any $E \in S(\alpha) \backslash A(\delta)$ and sufficiently small difference $\left|\alpha-\alpha^{\prime}\right|<h(\alpha, \delta)$ we can find $E^{\prime} \in S\left(\alpha^{\prime}\right)$ such that

$$
\left|E^{\prime}-E\right|<c(\alpha, \delta)\left|\left(\alpha-\alpha^{\prime}\right) \ln ^{3}\right| \alpha-\alpha^{\prime}|| .
$$

The constant $c$ is independent of $E, E^{\prime}, \alpha^{\prime}$.

\section{Remarks.}

1. The exponent 3 in (2.15) can be replaced by $2+\epsilon$ with any $\epsilon>0$, as the reader can easily verify starting with equation (2.5). For the almost Mathieu case, 3 can be replaced by 1 .

2. A similar statement (with 3 replaced by a higher power) holds under a standard Diophantine condition on $\alpha$.

3. This statement can be made uniform in $E \in S$ if we require $\gamma(E)$ to be bounded away from 0 (as in the case of the almost Mathieu operator) and use the uniform version of Lemma 2 .

Proof. As in $[6,3,16,13]$, for a given $E \in S(\alpha)$ we construct an approximate eigenvector for $H_{\alpha}$. In order to obtain almost-Lipschitz continuity we need the error of approximation to be exponentially small, as in [13].

Fix $\alpha \in \Omega$. Let $G=\{E \in S: \gamma(E)<g\}$ for some $g>0$. Then $|G| \rightarrow 0$ as $g \rightarrow$ 0 . Let $g$ be such that $|G|<\delta / 2$. Choose positive $\varepsilon^{\prime}$, $\varepsilon^{\prime \prime}$ so that $\varepsilon^{\prime}+\varepsilon^{\prime \prime} \leq g / 16$. Set $A(\delta)=F\left(\frac{\delta}{2}, \varepsilon^{\prime \prime}\right) \cup G$. Fix $E \in S \backslash A(\delta)$. Set $K=\left\{k \in M, k>2 k_{2}\left(\delta, \alpha, \varepsilon^{\prime \prime}\right)+3\right\}$ with $k_{2}$ from Lemma 2 , and $M$ defined in the proof of Lemma 1.

For any $E_{0} \in S$ let $G(x, y)$ be the matrix elements of $G=\left(\left(H-E_{0}\right)_{\left[x_{1}, x_{2}\right]}\right)^{-1}$. Using Cramer's rule and (2.7) at the last step, we obtain:

$$
\begin{gathered}
\left|G\left(x, x_{1}\right)\right|<\frac{\left|P_{x_{2}-x}\left(e^{2 \pi i \theta_{x+1}}, E_{0}\right)\right|+e^{-d k^{2}}}{\left|P_{k}\left(e^{2 \pi i \theta_{x_{1}}}, E_{0}\right)\right|-e^{-d k^{2}}}, \\
\left|G\left(x, x_{2}\right)\right|<\frac{\left|P_{x-x_{1}}\left(e^{2 \pi i \theta_{x_{1}}}, E_{0}\right)\right|+e^{-d k^{2}}}{\left|P_{k}\left(e^{2 \pi i \theta_{x_{1}}}, E_{0}\right)\right|-e^{-d k^{2}}},
\end{gathered}
$$

where $k=x_{2}-x_{1}+1>k_{1}$. 
We shall now fix $x_{1}, k$, and $E_{0}$. First take a $k \in K$. By Lemma 2, $\left|P_{[(k+j) / 2]}(z, E)\right|<\exp \left(\gamma(E)+\varepsilon^{\prime \prime}\right)(k / 2+1)$ for $j=-3,-2, \ldots, 2$, all $z$. We can find a neighborhood of $E, r_{k}(E)$ of diameter smaller than $\exp (-k g / 4)$, so that for any $E^{\prime \prime} \in r_{k}(E)$ we have for all $z(|z|=1):\left|P_{[(k+j) / 2]}\left(E^{\prime \prime}\right)\right|<$ $\left|P_{[(k+j) / 2]}(E)\right| \exp \left(\varepsilon^{\prime \prime}(k / 2+1)\right)$ and $\left|P_{k}\left(E^{\prime \prime}\right)\right|>\left|P_{k}(E)\right| \exp \left(-\varepsilon^{\prime} k\right)$. Now take a generalized eigenvalue $E_{0} \in r_{k}(E)$. Let $\psi$ be the corresponding generalized eigenfunction, that is a formal solution to the equation $H \psi=E_{0} \psi$ with $|\psi(x)|<\widetilde{C}(|x|+1)$ for all $x$. By $[18,7]$ the set of $E_{0}$ which admit such solutions is dense in the spectrum. There holds:

$$
\max _{x} \frac{|\psi(x)|}{|x|+1}=\frac{\left|\psi\left(x_{\max }\right)\right|}{\left|x_{\max }\right|+1}=R<\infty
$$

for some point $x_{\max }$. We normalize $\psi$ so that $R=1$.

As is easily seen by considering $\left(H-E_{0}\right)_{\left[x_{1}, x_{2}\right]} \psi$, we have for $x \in\left[x_{1}, x_{2}\right]$ :

$$
-\psi(x)=G\left(x, x_{1}\right) \psi\left(x_{1}-1\right)+G\left(x, x_{2}\right) \psi\left(x_{2}+1\right) .
$$

Define the interval $I=\left[x_{\max }-k-C k^{3}, x_{\max }-k\right]$, where $C=C\left(\varepsilon^{\prime}, \alpha\right)$ is the constant from Lemma 1. Then $|I|=C k^{3}$, and in view of Lemma 1 , we can find $x_{1}=$ $m$ in $I$ (which fixes position of the interval $\left[x_{1}, x_{2}\right]$ ) such that $\left|P_{k}\left(e^{2 \pi i \theta_{m}}, E\right)\right| \geq$ $\exp \left(\gamma(E)-\varepsilon^{\prime}\right) k$ and hence $\left|P_{k}\left(e^{2 \pi i \theta_{m}}, E_{0}\right)\right|>\exp \left(\gamma(E)-2 \varepsilon^{\prime}\right) k$. We now evaluate $\psi(x)$ at the midpoint of the interval $\left[x_{1}, x_{2}\right]: x_{0}=x_{1}+[(k-1) / 2]$ and at its nearest neighbors. Using Lemma 1 to evaluate the denominator in (2.16), we obtain $\left|G\left(x_{0}, x_{1}\right)\right|<2 \exp ((-\gamma(E)+\varepsilon) k / 2+\gamma(E)+\varepsilon), \varepsilon=4\left(\varepsilon^{\prime}+\varepsilon^{\prime \prime}\right)$ and the same estimate for $G\left(x_{0}, x_{2}\right)$ for sufficiently large $k \in K$ (depending on $d$ ). Applying (2.18), we get

$$
\left|\psi\left(x_{0}-1\right)\right|,\left|\psi\left(x_{0}\right)\right|<4 e^{-(g-\varepsilon) k / 2+D+\varepsilon}\left(\left|x_{\max }\right|+(C+2) k^{3}\right)
$$

for sufficiently large $k \in K$.

Now let $I^{\prime}=\left[x_{\max }+k, x_{\max }+k+C k^{3}\right]$. Similarly, we choose an interval $\left[x_{1}^{\prime}, x_{2}^{\prime}\right]$ so that at $x_{1}^{\prime} \in I^{\prime}$ we have a lower bound of Lemma 1 . We apply again Lemmas 1 and 2 to get the same estimate $(2.19)$ for $\psi\left(x_{0}^{\prime}\right)$ and $\psi\left(x_{0}^{\prime}+1\right)$ at the midpoint $x_{0}^{\prime}$ of $\left[x_{1}^{\prime}, x_{2}^{\prime}\right]$ and at $x_{0}^{\prime}+1$. By construction, $L=\left|x_{0}-x_{0}^{\prime}\right|$ satisfies $k<L<2(C+1) k^{3}$. Set

$$
\psi_{L}(x)= \begin{cases}\psi(x), & x \in\left[x_{0}, x_{0}^{\prime}\right] \\ 0, & \text { otherwise. }\end{cases}
$$

and $\phi_{L}(x)=\psi_{L}(x) /\left\|\psi_{L}\right\|$. Since $x_{\max } \in\left[x_{0}, x_{0}^{\prime}\right]$, we have $\left\|\psi_{L}\right\|>\left|x_{\max }\right|+1$. Hence

$$
\begin{aligned}
\frac{\left|\psi\left(x_{0}-1\right)\right|}{|| \psi_{L} \|},\left|\phi_{L}\left(x_{0}\right)\right|, \frac{\left|\psi\left(x_{0}^{\prime}+1\right)\right|}{|| \psi_{L}||},\left|\phi_{L}\left(x_{0}^{\prime}\right)\right|< \\
4 e^{-(g-\varepsilon) k / 2+D+\varepsilon} \frac{\left|x_{\max }\right|+(C+2) k^{3}}{\left|x_{\max }\right|+1}<(C+2) e^{-(g-2 \varepsilon) k / 2}
\end{aligned}
$$

for $k \in K, k>K_{0}$, where $K_{0}(\varepsilon, D, d)$ is sufficiently large. 
By the variational principle, there exists a point $E^{\prime}$ in the spectrum of $H_{\alpha^{\prime}, \theta^{\prime}}$ (here $\left.\theta^{\prime}=\left(\alpha-\alpha^{\prime}\right) x_{\max }+\theta\right)$ such that

$$
\begin{aligned}
& \left|E^{\prime}-E\right| \leq\left\|\left(H_{\alpha^{\prime}, \theta^{\prime}}-E\right) \phi_{L}\right\| \leq \\
& \left\|\left(H_{\alpha^{\prime}, \theta^{\prime}}-H_{\alpha, \theta}\right) \phi_{L}\right\|+\left\|\left(H_{\alpha, \theta}-E_{0}\right) \phi_{L}\right\|+\left|E-E_{0}\right|< \\
& C^{\prime}\left|\alpha-\alpha^{\prime}\right| L+4(C+2) e^{-(g-2 \varepsilon) k / 2}+e^{-k g / 4}< \\
& C^{\prime} 2(C+1)\left|\alpha-\alpha^{\prime}\right| k^{3}+4(C+3) e^{-g k / 4},
\end{aligned}
$$

where we applied the estimate $\left|f(\alpha n+\theta)-f\left(\alpha^{\prime} n+\theta^{\prime}\right)\right|<C^{\prime}\left|\alpha-\alpha^{\prime}\right| L$ with some $C^{\prime}>0$ and used that, by our choice of parameters, $2 \varepsilon \leq g / 2$. Let $\alpha^{\prime}$ be sufficiently close to $\alpha$, so that $k=\left[4|\ln | \alpha-\alpha^{\prime}|| / g\right]$ (or $k \pm 1$ ) is in $K$ and larger than $K_{0}$ and $|\ln | \alpha-\alpha^{\prime}||$ is sufficiently large depending on values of $C, C^{\prime}$, and $g$. Then we obtain from (2.21) the statement of Theorem 3 with $c(\alpha, \delta)=$ $2^{7} C^{\prime}(C+1) / g^{3}+1$.

\section{Proof of Theorem 1}

As we noted in the introduction, the theorem for $\alpha \notin \Omega$ is easy to prove following [3, 16]. Take $\alpha \in \Omega$ and consider the sequence of its canonical rational approximants $p_{n} / q_{n}$. Because of continuity in $\theta$, the set $S\left(p_{n} / q_{n}\right)$ consists of at most $q_{n}$ disjoint intervals, say $S\left(p_{n} / q_{n}\right)=\cup_{i=1}^{q_{n}^{\prime}}\left[a_{i}, b_{i}\right], q_{n}^{\prime} \leq q_{n}$. Given the continuity result of Theorem 3 , the proof of Theorem 1 differs from the corresponding analysis for the almost Mathieu operator in [16] only in one detail. By $[19,17]$ we have $|S(\alpha)| \geq \lim \sup _{n \rightarrow \infty}\left|S\left(p_{n} / q_{n}\right)\right|$. We are going to show that $|S(\alpha)| \leq \liminf _{n \rightarrow \infty}\left|S\left(p_{n} / q_{n}\right)\right|$ when $\gamma(E)>0$ for a.e. E. For all $n$ sufficiently large (such that $\left|p_{n} / q_{n}-\alpha\right|<h(\alpha, \delta)$ ), Theorem 3 says that, except perhaps for the points belonging to $A(\delta)$, all the other points in the spectrum of $H_{\alpha}$ are necessarily inside the set $\left(\alpha^{\prime}=p_{n} / q_{n}\right)$

$$
\cup_{i=1}^{q^{\prime} n}\left[a_{i}-c\left|\left(\alpha-\alpha^{\prime}\right) \ln ^{3}\right| \alpha-\alpha^{\prime}||, b_{i}+c\left|\left(\alpha-\alpha^{\prime}\right) \ln ^{3}\right| \alpha-\alpha^{\prime}||\right] .
$$

Therefore,

$$
|S(\alpha)|<\left|S\left(p_{n} / q_{n}\right)\right|+2 c q_{n}\left|\left(\alpha-p_{n} / q_{n}\right) \ln ^{3}\right| \alpha-p_{n} / q_{n}||+\delta
$$

As $n \rightarrow \infty$, the second addend at the right hand side tends to 0 for any irrational $\alpha$ because of (2.10). The constant $\delta>0$ can be taken arbitrary small by Theorem 3. Hence, $|S(\alpha)|=\lim _{n \rightarrow \infty}\left|S\left(p_{n} / q_{n}\right)\right|$.

\section{Acknowledgements}

We would like to acknowledge the support of the NSF grant DMS-9800860. The first author was also supported under NSF grant DMS-0070755, and the second author by Sonderforschungsbereich 288. The second author is grateful to S. Jitomirskaya and A. Klein for their hospitality in the University of California, Irvine, where part of this work was written. 


\section{References}

[1] S. Aubry, G. André, Analyticity breaking and Anderson localisation in incommensurate lattices, Ann. Israel Phys. Soc. 3 (1980), 133-164.

[2] J. E. Avron, B. Simon, Almost periodic Schrödinger operators. II. The density of states, Duke Math J. 50 (1983), 369-391.

[3] J. Avron, P. van Mouche, B. Simon, On the measure of the spectrum for the almost Mathieu operator, Comm. Math. Phys. 132 (1990), 103-118.

[4] J. Bourgain, M. Goldstein, On nonperturbative localization with quasi-periodic potential, Ann. of Math. (2) 152 (2000), 835-879.

[5] J. Bourgain, S. Jitomirskaya, Continuity of the Lyapunov exponent for quasiperiodic operators with analytic potential, preprint.

[6] M.-D. Choi, G. A. Elliott, N. Yui, Gauss polynomials and the rotation algebra, Invent. Math. 99 (1990), 225-246.

[7] H. L. Cycon, R. G. Froese, W. Kirsch, B. Simon, Schrödinger operators with application to quantum mechanics and global geometry, Springer-Verlag, Berlin, 1987.

[8] G. A. Elliott, Gaps in the spectrum of an almost periodic Schrödinger operator, C.R. Math. Rep. Acad. Sci. Canada 4 (1982), 255-259.

[9] A. Furman, On the multiplicative ergodic theorem for uniquely ergodic systems, Ann. Inst. H. Poincaré Probab. Statist. 33 (1997), 797-815.

[10] M. Goldstein, W. Schlag, Hölder continuity of the integrated density of states for quasiperiodic Schrödinger equations and averages of shifts of subharmonic functions, Ann. Math., Ann. of Math. (2) 154 (2001), 155-203.

[11] B. Helffer, J. Sjostrand, Semiclassical analysis for Harper's equation. III. Cantor structure of the spectrum, Mém. Soc. Math. France (N.S.) 39 (1989), 1-124.

[12] S. Ya. Jitomirskaya, Metal-insulator transition for the almost Mathieu operator, Ann. of Math. (2) 150 (1999), 1159-1175.

[13] S. Jitomirskaya, Y. Last, Anderson localization for the almost Mathieu equation. III. Semi-uniform localization, continuity of gaps, and measure of the spectrum, Comm. Math. Phys. 195 (1998), 1-14.

[14] , Power law subordinacy and singular spectra. II. Line operators, Comm. Math. Phys. 211 (2000), 643-658.

[15] A. Ya. Khintchine, Continued fractions, P. Noordhoff, Ltd., Groningen 1963.

[16] Y. Last, A relation between a.c. spectrum of ergodic Jacobi matrices and the spectra of periodic approximants, Comm. Math. Phys. 151 (1993), 183-192.

[17] _ Zero measure spectrum for the almost Mathieu operator, Comm. Math. Phys. 164 (1994), 421-432.

[18] I. E. Shnol, On the behaviour of eigenfunctions, Dokl. Akad. Nauk SSSR 44 (1954), 389392.

[19] D. J. Thouless, Bandwidths for a quasi-periodic tight-binding model, Phys. Rev. B 28 (1983), 4272-4276.

Department of Mathematics, University of California, Irvine, CA 92697, U.S.A.

E-mail address: szhitomi@math.uci.edu

Technische Universität Berlin, Institut für Mathematik MA 7-2 Strasse des 17. Juni 136, D-10623, Berlin, Germany.

E-mail address: ivk@math.tu-berlin.de 